\title{
Nonlinear diffusion and image contour enhancement
}

\author{
G. I. BARENBLATT ${ }^{\dagger}$ \\ Department of Mathematics and Lawrence National Laboratory, \\ University of California at Berkeley, \\ Berkeley, CA 94720-3840, USA
}

AND

J. L. VÁZQUEZ

Departamento de Matemáticas, Universidad Autónoma de Madrid, 28046 Madrid, Spain

[Received 5 April 2003 and in revised form 24 November 2003]

The theory of degenerate parabolic equations of the forms

$$
u_{t}=\left(\Phi\left(u_{x}\right)\right)_{x} \quad \text { and } \quad v_{t}=(\Phi(v))_{x x}
$$

is used to analyze the process of contour enhancement in image processing, based on the evolution model of Sethian and Malladi. The problem is studied in the framework of nonlinear diffusion equations. It turns out that the standard initial value problem solved in this theory does not fit the present application since it does not produce image concentration. Due to the degenerate character of the diffusivity at high gradient values, a new free boundary problem with singular boundary data can be introduced, and it can be solved by means of a nontrivial problem transformation, thus leading to a new type of solutions that fit the desired concentration requirements. The asymptotic convergence to a sharp front is established and rates calculated.

Keywords: Nonlinear diffusion; image enhancement; degenerate parabolic equations; singular solutions; free boundaries.

2000 Mathematics Subject Classification: 35K55, 35K65.

\section{Introduction. A model for contour enhancement}

This paper is devoted to the behaviour of the solutions of some degenerate parabolic equations with moving boundaries which appear in describing the technique of contour enhancement in image processing. Indeed, computer vision has become in recent decades a mathematical discipline which relies on the differential-geometric approach. More specifically, an appropriate technique of image processing consists of formulating a partial differential equation of evolution type for the image intensity, $u(x, y)$. This function, also called the grey level, takes values in the interval $0 \leqslant u \leqslant 1$ and is defined on a two-dimensional image domain, $\Omega$. The usual evolution model leads to a nonlinear equation of parabolic type, possibly degenerate or singular. The nonlinearity is created by the law relating the image intensity flux to the image intensity.

\footnotetext{
Email: gib@math.uberkeley.edu

Email: juanluis.vazquez@uam.es
} 
It has been observed by Perona and Malik [44], 1990, that for a suitable choice of this fundamental nonlinearity there appears an effect of enhancement of image edges that is of strong interest in the application to processing, denoising and recognition of images. The Perona-Malik Anisotropic Diffusion model has had a deep influence in the field, being at the source of many later developments. The model proposed by Malladi and Sethian [40] leads (after proper scaling) to the following equation for the image intensity:

$$
u_{t}=\left(1+|D u|^{2}\right)^{1 / 2} \kappa,
$$

where $D u$ denotes the spatial gradient of $u$, and $\kappa$ denotes the curvature of the surface $z=u(x, y)$. The equation represents movement by curvature (curvature flow) and can be written as

$$
u_{t}=\frac{\left(1+u_{y}^{2}\right) u_{x x}-2 u_{x} u_{y} u_{x y}+\left(1+u_{x}^{2}\right) u_{y y}}{1+u_{x}^{2}+u_{y}^{2}} .
$$

We consider here the more general flow given by the equation $u_{t}=\left(1+|D u|^{2}\right)^{(1+p) / 2} \kappa$, where $p$ is a constant parameter. In other words, we study the equations

$$
u_{t}=\frac{\left(1+u_{y}^{2}\right) u_{x x}-2 u_{x} u_{y} u_{x y}+\left(1+u_{x}^{2}\right) u_{y y}}{\left(1+u_{x}^{2}+u_{y}^{2}\right)^{1+\alpha}}
$$

with parameter $\alpha=-p / 2$. Along with the former case $p=\alpha=0$, the case $p=-2, \alpha=1$ has also attracted the attention of researchers (Beltrami flow, cf. Sochen et al. [50]).

The asymptotic and numerical treatment of these models done in the papers [40, 50] shows the enhancement of the intensity contrasts by formation of regions of large intensity gradients, i.e., the normal component of the image intensity gradient becomes quite large. This phenomenon allowed [10] to suggest the existence of a boundary layer where large gradients concentrate and to focus on this boundary layer where a further simplification of the model is possible. Arguing locally around a sharp gradient point and choosing the $x$-axis as the direction normal to the boundary layer or front, we may disregard the effect of $y$ derivatives with respect to the $x$ derivatives in $(1.3)$. In this way we get the reduced equation, which is just the one-dimensional version of (1.3),

$$
u_{t}=\frac{u_{x x}}{\left(1+u_{x}^{2}\right)^{1+\alpha}}
$$

where we have neglected $u_{y}, u_{y y}$. Different dimensional constants appear in the model, but they have been scaled to unity here without loss of generality. The mathematical problem consists in solving this equation with suitable boundary data, namely, $u=0$ on the left-hand side of the contour and $u=1$ on the right-hand side (be that a finite or an infinite distance), and initial conditions

$$
u(x, 0)=u_{0}(x)
$$

satisfying $0<u_{0}<1$ and $u_{0}^{\prime}>0$ in an interval $I=(a, b)$ and constant values otherwise, zero to the left, 1 to the right. As was pointed out in [10], the phenomenon of gradient enhancement takes place in this model (in a proper setting) for all $\alpha \geqslant 0$ : the spatial gradient of the solutions, $u_{x}$, increases with time, and its support shrinks. Indeed, we show below that such behaviour can be observed in the larger exponent range $\alpha>-1 / 2$. The conditions on $u_{0}$ can be relaxed, but then problems arise. Thus, the less stringent size restriction $0 \leqslant u_{0} \leqslant 1$ or the lack of monotonicity create interesting alternatives, that will also be briefly discussed. 
Let us remark that this is not the only model which uses nonlinear diffusion equations for image processing and enhancement. We refer for alternatives to the works of Alvarez, Lions and Morel [1] and Caselles et al. [26], where further references are found. Let us point out that in [1] a fundamentally different assumption is made, namely that the flux is perpendicular to the gradient, and the basic equation is substantially different from the class (1.3) treated here. In the paper by Osher and Rudin [42] a model of image enhancement was considered, leading to an evolution equation of the form (in the $1 \mathrm{D}$ case)

$$
u_{t}=-\left|u_{x}\right| F\left(u_{x x}\right) .
$$

The idea is that the nonlinear evolution equation acts as a shock filter (see also [48, 33]). The processed image is piecewise smooth and nonoscillatory. Model $(1.5)$ is also substantially different from the class of models considered in the present paper. In particular, as is shown in detail in [42], the values of the image intensity remain constant at the points where $u_{x}=0$. On the other hand, we mention that the mathematical difficulties of the original model by Perona and Malik have been further investigated by several authors, like [37] and [59]. Summing up, the possible application of the techniques of nonlinear diffusion to image processing is a very active concern with many issues being discussed in the literature (cf. e.g. [39, [58]).

Outline of the paper. After an introduction to the mathematical facts on enhancement contained in [10], we revise the needed concepts of nonlinear diffusion in 1D in Section 3 and derive the differentiated model, a porous medium type equation, in Section 4. Section 5 is devoted to the standard theory for the two diffusion models we have introduced, which leads to so-called solutions of Type I: they are purely diffusive and exhibit no contour enhancement.

The existence and uniqueness of solutions with contracting interfaces, the goal of the paper, is discussed in Sections 6 and 7, and the asymptotic behaviour in Section 8. Two short sections follow: Section 9 contains asymptotic estimates for general nonlinearities and Section 10 briefly presents numerical experiments. A final Section 11 contains comments on different aspects of the work and new directions.

\section{Asymptotic self-similarity and enhancement}

Evolution equations like (1.4) and many other variants have been studied and are known in the literature under the general name of nonlinear parabolic equations of diffusion type, or nonlinear diffusion equations for short. They are typically used in describing processes of mass diffusion or thermal propagation. A quite general one-dimensional form popular among PDE experts is

$$
u_{t}=\left(a\left(x, t, u, u_{x}\right)\right)_{x}
$$

with suitable conditions to make it parabolic, at least in a formal sense, like $\partial a / \partial u_{x}>0$. However, many practical applications (as in the present case) involve functions $a$ for which $\partial a / \partial u_{x} \geqslant 0$, but the values zero or infinity can also be taken, and then the equations are known as degenerate parabolic or singular parabolic resp. (cf. [28, 36]). In any case, a general feature of this wide class of equations is their diffusive character, which roughly means the spreading of the level sets of the solutions as time advances. This property goes squarely against the desired enhancement, therefore an extra mechanism must be present if enhancement is to occur. We recall that in the Perona-Malik model this mechanism was negative diffusion. 
In the range of exponents $\alpha \geqslant 0$, equation (1.4) falls into the class of degenerate parabolic equations with degeneracy at $u_{x}=\infty$, precisely the limit value which is of concern in contour enhancement. An investigation of the phenomenon of gradient enhancement in equation [1.4] is performed in [10] by relating it to the convergence toward self-similar asymptotics of an approximate model. It goes as follows: the observed evolution of the solutions towards a configuration with large gradients makes it plausible to further simplify the expression $1+u_{x}^{2}$ in 1.4 into $u_{x}^{2}$, so that the relevant reduced equation becomes

$$
u_{t}=u_{x}^{-2(1+\alpha)} u_{x x}
$$

It is further observed that self-similar solutions for this equation with end-levels $u=0$ and $u=1$ exist for all $\alpha>0$ and exhibit the similarity form

$$
u(x, t)=F(\xi), \quad \xi=\left(x-x_{0}\right)\left(t+t_{0}\right)^{1 / 2 \alpha},
$$

where $x_{0}$ and $t_{0}$ are parameters to be fixed, and the profile $F(\xi)$ is an increasing function joining the levels $F=0$ at a finite distance $\xi=-c<0$ to the level $F=1$ at $\xi=c$. At these levels, which are taken at a finite distance $\xi= \pm c$, the gradients are infinite (actually, the analysis in [10] deals with decreasing profiles joining $u=1$ to $u=0$ but these two problems are obviously equivalent after a mirror symmetry. We have chosen the increasing option to avoid chasing around many minus signs). The scaling implies that

$$
u_{x}(x, t)=\left(t+t_{0}\right)^{1 / 2 \alpha} F^{\prime}(\xi), \quad \xi=\left(x-x_{0}\right)\left(t+t_{0}\right)^{1 / 2 \alpha},
$$

which shows that the solution is concentrated in an increasingly narrower strip

$$
S=\left\{(x, t):\left|x-x_{0}\right| \leqslant c\left(t+t_{0}\right)^{-1 / 2 \alpha}\right\}
$$

with gradients that diverge like $t^{1 / 2 \alpha}$ as $t \rightarrow \infty$. Let us remark that the asymptotic divergence of the gradients is known to be exponential for $\alpha=0$, but the form is not self-similar of the same type, as we will see below. An important feature to be remembered of these solutions, along with the divergence as $t \rightarrow \infty$, is the infinite gradient condition at the endpoints of the domain of definition. On the other hand, these self-similar solutions represent the intermediate asymptotics of the problem with more general data. This is demonstrated numerically in [10] when infinite flux data are imposed at moving endpoints located at finite distance.

With these preliminaries we are ready to attack the construction and analysis of solutions with steep fronts for a general class of equations which includes (2.1) and (1.4) and is natural for our application, and with the general data mentioned in the Introduction. We use the theory of nonlinear diffusion equations which has been strongly developed in the last decades. It turns out that the standard initial value problem solved in this theory does not suit our model, since it leads to dispersion instead of concentration of the image. A new free boundary problem with singular boundary data is then introduced and solved by means of a number of nontrivial problem transformations available for nonlinear diffusion problems, a subject that is developed in detail in [55]. The concentration property of these solutions is possible thanks to the degenerate character of the diffusivity for high gradient values. This is related to work on nonlinear diffusive models with free boundaries as described by a number of authors, like [19, 21, 34, 35], but the main mathematical feature of our study is the presence of infinite gradients at the moving boundary.

Our theory below covers existence and uniqueness of solutions of suitable problems, existence and behaviour of the bounding interfaces, and large time behaviour. In particular, we obtain the rates of convergence of the interfaces, an important question for image enhancement. 


\section{Nonlinear diffusion equations}

As we have just said, we are going to construct a mathematical theory for the one-dimensional evolution problem with initial and boundary conditions of the type mentioned above, but replacing the special class of equations (1.4) by the wider class of nonlinear diffusion equations of the form

$$
u_{t}=\left(\Phi\left(u_{x}\right)\right)_{x}
$$

The nonlinear function $\Phi$ expresses the dependence of the image flux on the gradient of the image intensity. It can be called the flux law of our process, or also the constitutive function. In this general setting, $\Phi$ can be any increasing real function defined in a suitable interval of gradients. We will assume for convenience that

$\left(\mathbf{H}_{1}\right) \Phi(s)$ is $C^{\infty}$ smooth and strictly increasing in the intervals $0<s<\infty$ and $-\infty<s<0$, allowing for a quite arbitrary behaviour as $s \rightarrow 0$ or $|s| \rightarrow \infty$.

In our opinion, this level of generality instead of obscuring the problem makes more apparent the relation between nonlinear diffusion and contour enhancement. Slightly more general assumptions can be made on $\Phi$, but they are unessential for our present purposes (cf. Section 11).

We want to characterize the class of those $\Phi$ for which we can construct solutions with gradientenhancement. We also want to describe the rates at which the formation of steep profiles takes place, thus justifying and extending the results of [10] to the actual equations like (1.4), and to general data. The mathematical problem we pose consists in solving the nonlinear parabolic equation 3.1) with initial conditions

$$
u(x, 0)=u_{0}(x)
$$

satisfying $0 \leqslant u_{0} \leqslant 1$ and other suitable conditions (see below), and boundary data $u=0$ on the left-hand side of the contour and $u=1$ on the right-hand side. Regarding the initial data, we remark that we are mainly interested in monotone solutions, i.e., $u_{x} \geqslant 0$, for equation (1.4), and this condition will naturally follow from a similar monotonicity condition on the initial data. As for the boundary conditions, it turns out that, depending on the form of $\Phi$, the boundary can be chosen to be located either at infinity or at a finite distance. This latter case will be the one of interest for us, and then the problem must be properly posed as a free boundary problem.

Let us recall the simplest examples of $\Phi$ and some of the difficulties we will encounter. Indeed, a quite important and simple example is the power function, that we write as $\Phi(s)=(1 / m) s^{m}$, defined for $s \geqslant 0$, so that it agrees with 2.1) with $m=-1-2 \alpha$ since $\Phi^{\prime}(s)=s^{m-1}$. There is in principle no reason to restrict the generality of the exponent $m$ in the mathematical treatment to follow, and this will lead to quite different behaviour types inside this family. The case $m=0$ is included in the form $\Phi(s)=\log (s)$, i.e., $\Phi^{\prime}(s)=1 / s$. Note that in the cases with $m<0$ the function $\Phi$ is negative, but the important quantity for the parabolic character of the equation, i.e., $\Phi^{\prime}\left(u_{x}\right)$, is always positive. Finally, we note that equation 1.4$)$ corresponds to $\Phi^{\prime}(s)=\left(1+s^{2}\right)^{-(1+\alpha)}$; it degenerates as $s \rightarrow \infty$ in the prescribed range $\alpha \geqslant 0$, even if $\alpha>-1$, but the equation is is perfectly parabolic in regions of bounded $u_{x}$.

\subsection{Nonmonotone solutions}

The above statements are made on the assumption that the solutions are monotone, $u_{x} \geqslant 0$, which is not unjustified in our problem setting but restricts the mathematical generality. When considering 
nonmonotone solutions it is customary in the nonlinear diffusion literature to extend the power nonlinearity to arguments $s=u_{x}<0$ in the simplest symmetric way:

$$
\Phi(s)=\frac{1}{m}|s|^{m-1} s,
$$

which gives $\Phi^{\prime}(s)=|s|^{m-1}$, always nonnegative. This definition poses no problem when $m>0$ and solutions with changing sign exist corresponding to initial data with the same property. However, we are interested in exponents $m<0$ (so-called very fast diffusion in the literature) where $\Phi^{\prime}$ is singular at $s=0$, and the whole function $\Phi$ is no more monotone. The difficulty has been studied in [47] leading to the consequence that solutions with changing sign do not exist, even in the weak sense when $m \leqslant 0$. This is a consequence of the singularity of the equation at the level $u_{x}=0$ and does not affect equation (1.4). Due to this obstruction, we will concentrate here on problems with monotone solutions (but see comment in the final section).

\section{Second formulation as a nonlinear diffusion equation}

If we formally differentiate equation 3.1 with respect to $x$ and put $v=u_{x}$, we obtain the equation satisfied by the image intensity gradient:

$$
v_{t}=\Phi(v)_{x x}
$$

which is usually called the nonlinear filtration equation, NLFE, and is the most standard class of nonlinear diffusion equations studied in the literature. We can call it in this context the "differentiated equation". Conversely, we can recover the $u$ formulation from a solution $v(x, t)$ for (4.1) by means of the rule

$$
u(x, t)=c+\int_{\Gamma}\left(v \mathrm{~d} x+\Phi(v)_{x} \mathrm{~d} t\right)
$$

integrated along any curve $\Gamma$ in the domain of definition of $v$ which joins a fixed point $\left(x_{0}, t_{0}\right)$ to the generic point $(x, y)$ (cf. [46, 55]). The constant $c$ is the value of $u$ at $\left(x_{0}, t_{0}\right)$ to be chosen at will in principle. The calculation is justified for smooth solutions $v$ and smooth $\Phi$, but holds in a much wider context.

Monotone solutions $u$ for equation (1.4) translate into nonnegative solutions $v$ for equation (4.1), and conversely. In this context, $u$ is usually viewed as the mass function for $v$, since when we take $\Gamma$ to be a segment of the line $t=t_{0}$, formula (4.2) becomes

$$
u\left(x, t_{0}\right)-u\left(x_{0}, t_{0}\right)=\int_{x_{0}}^{x} v\left(y, t_{0}\right) \mathrm{d} y .
$$

The phenomenon of gradient enhancement can then be translated into usual diffusion parlance as mass concentration. We will keep in what follows the denomination intensity or image intensity for the solution $u$ of equation (3.1), and we will view the solution $v=u_{x}$ of (4.1) as the intensity gradient. In that context $\Phi\left(u_{x}\right)=\Phi(v)$ is the intensity flux. Finally, $\Phi^{\prime}\left(u_{x}\right)=\Phi^{\prime}(v)$ is the diffusivity. 


\section{Solutions of Type I}

We now try to apply the more standard theory of nonlinear diffusion equation to solve the evolution problem motivated by the application in the Introduction. We show next that this implementation can be performed in a rather standard way for a wide class of $\Phi$ 's, but such a process gives an evolution with no concentration effect, hence useless for our purposes in image processing. The reader already familiar with diffusion theory may check the contents of Theorem 5.1 and skip the rest of this section.

\subsection{Simplest setting}

Generalizing the well known properties of the heat equation (the choice $\Phi(s)=s$ ), we consider first the case where $\Phi$ is a $C^{1}$ function and $\Phi^{\prime}$ does not vanish. We pose the Cauchy problem for equation (3.1) on the whole line $x \in \mathbb{R}$ for $t>0$ with bounded initial data $0 \leqslant u_{0} \leqslant 1$, and get a unique smooth solution $u(x, t)$ defined in $Q=\mathbb{R} \times(0, \infty)$ and such that $0<u<1, u$ is smooth for all $x \in \mathbb{R}$ and $t \geqslant \tau>0$. We can also work with equation 4.1 : then, if $v_{0}=u_{0, x}$ is locally integrable (a Radon measure will also do) and such that

$$
\int_{\mathbb{R}} v_{0}(x) \mathrm{d} x=1
$$

the solution $v(x, t)$ is smooth in $Q$ and satisfies the same type of integrability condition, $\int_{\mathbb{R}} v(x, t) \mathrm{d} x=1$. For solutions $v \geqslant 0$ this is called conservation of mass. Using formula 4.2 we obtain a smooth solution $u(x, t)$ in $Q$ such that the initial condition

$$
u(x, 0)=u_{0}(x)=\int_{-\infty}^{x} v_{0}(y) \mathrm{d} y
$$

holds, as well as the end conditions

$$
\lim _{x \rightarrow-\infty} u(x, t)=0, \quad \lim _{x \rightarrow \infty} u(x, t)=1,
$$

hold locally uniformly in time. On the other hand, $v_{0} \geqslant 0$ implies that $u_{x}=v \geqslant 0$, which by the Strong Maximum Principle implies $u_{x}>0$. It follows that $0<u(x, t)<1$ in $Q$. Note that, due to the smoothness condition on $\Phi$, these solutions are smooth in $(x, t)$ for all $x \in \mathbb{R}$ and $t>0$, even if the initial data are not.

\subsection{Dispersion of solutions with time}

At first glance, the type of solution we have constructed seems to solve our image problem. However, it lacks a basic ingredient, i.e., the eventual concentration of intensity gradients. On the contrary, if we consider the heat equation $u_{t}=u_{x x}$, the solutions, which can be expressed in terms of error functions, spread in time and its gradients go to zero. For instance, if $v_{0}=u_{0, x}$ is integrable, then $v(x, t)$ goes to zero as $t \rightarrow \infty$ at a rate of the order of $t^{-1 / 2}$, and takes on a Gaussian space shape,

$$
v(x, t) t^{1 / 2} \sim \exp \left(-x^{2} / 4 t\right) .
$$

And a similar result (with possibly different rates) applies to more general data $u_{0}$ having definite limits at $\pm \infty$, and to all the functions $\Phi$ of the above class. 


\subsection{Extension of Type I solutions to other $\Phi$}

Even if this is not the class of solutions we are looking for, we pursue a bit further the analysis, since it is the standard class found in studies of nonlinear diffusion or thermal propagation. The study will also serve for comparison with the "correct" solutions of Type II. However, the reader may choose to skip the rest of the section and proceed with Section 6 .

The class of constitutive functions $\Phi$ for which there exists a class of solutions $0 \leqslant u \leqslant 1$ with a dispersive character can be extended to include a quite general choice of $\Phi$ on the condition of allowing for a suitably generalized concept of solution. Thus, it is well known following Bénilan, Crandall and others [14,16] that we may take as $\Phi$ any continuous nondecreasing function and the Cauchy problem is then well-posed in the class of so-called mild solutions with $L^{1}(\mathbb{R})$ data. Actually, $\Phi$ can be allowed to be discontinuous but this will be of no particular interest here; on the contrary, we will keep the assumption of smoothness and strict monotonicity for $0<s<\infty$ for simplicity of presentation (and since it is satisfied in the application we are dealing with).

Let us comment on the main properties of the solutions for this class of equations. For the case of power nonlinearities $\Phi(s)=s^{m} / m, s \geqslant 0$, mentioned above, rather complete details are known, [6, 36, 52]. We can consider that $m$ is a real parameter, positive in principle but not necessarily as we will see. The definition is extended to solutions with negative values as in formula (3.3). Thus, for $m>0$ the equation $v_{t}=\left(v^{m}\right)_{x x}$ (or better, $v_{t}=\left(|v|^{m-1} v\right)_{x x}$ ) generates a positive semigroup of contractions in the space $L^{1}(\mathbb{R})$. In other words, for every $v_{0} \in L^{1}(\mathbb{R})$ there exists a unique function $v \in C\left([0, \infty) ; L^{1}(\mathbb{R})\right), v \geqslant 0$ with $v^{m} \in L_{\text {loc }}^{1}(Q)$, such that the equation is satisfied in the sense of distributions in $Q=\mathbb{R} \times(0, \infty)$, the initial data are taken in the $L^{1}(\mathbb{R})$ sense, and the map $v_{0} \mapsto v(\cdot, t)$ is an $L^{1}$-contraction. Moreover, the total mass is conserved;

$$
\int_{0}^{\infty} v(x, t) \mathrm{d} x=\int_{0}^{\infty} v_{0}(x) \mathrm{d} x .
$$

In our application we still have to impose the extra condition of total mass 1 , and then we recover the image intensity by means of the formula

$$
u(x, t)=\int_{-\infty}^{x} v\left(x^{\prime}, t\right) \mathrm{d} x^{\prime}
$$

and the intensity level goes from $u=0$ at minus infinity to $u=1$ at infinity. The asymptotic behaviour of these solutions has been carefully calculated in the literature (cf. e.g. [5, 31, 53]): solutions $v(\cdot, t)$ with finite mass go to zero uniformly as

$$
v(x, t) \sim t^{-\gamma}, \quad \gamma=\frac{1}{m+1},
$$

which in the notation of the Introduction means $\gamma=-1 / 2 \alpha$. Actually, the asymptotic rate comes from comparison with the source-type self-similar solutions [9] which take the form

$$
v(x, t)=t^{-\gamma} G\left(x t^{-\gamma}\right)
$$

for a certain symmetric profile function $G \in C_{b}(\mathbb{R})$ such that $G(\xi) \rightarrow 0$ as $\xi \rightarrow \infty$. This formula suggests that the behaviour will be maintained as long as $\gamma>0$, hence, as long as $m>-1$, and this turns out to be true (for the study when $-1<m \leqslant 0$ cf. [29]). Indeed, this exponent range is optimal since there are no solutions with finite integral for $m \leqslant-1$. 
Let us look a bit closer at the kind of initial data that we want to consider in the outmost generality. We recall that we want $u_{0}$ to be increasing and bounded between 0 and 1 . Hence, $v_{0}=u_{0}^{\prime}$ has to be defined and locally integrable, or at most be a measure, in an interval $(a, b)$ with possible divergence at the endpoints, but anyway with finite integral. Existence of solutions (with $v_{0}$ extended all of $\mathbb{R}$ with value 0 otherwise) offers no problem in all the range $m>-1$ and the solutions are bounded for all $t>0$. But large values will be the origin of the new class of solutions to discuss in the following sections.

By integration we obtain a solution of the problem

$$
\left(\mathrm{P}_{\mathrm{I}}\right) \begin{cases}u_{t}=\Phi\left(u_{x}\right)_{x} & \text { in } Q=\mathbb{R} \times(0, \infty), \\ u(x, 0)=u_{0}(x) & \text { for } x \in \mathbb{R}, \\ u(x, t) \geqslant 0, & \end{cases}
$$

where $u_{0}$ is any nondecreasing continuous real function joining the levels $u_{0}=0$ at $x=-\infty$ to $u_{0}=1$ at $x=\infty$. This is what we term solutions of Type I. Note that integration of the self-similar profile gives for $u$ the form

$$
u(x, t)=F\left(x t^{-\gamma}\right)
$$

where $F$ is a primitive of $G$ with $F(-\infty)=0, F(\infty)=M$ that we want to normalize to $M=1$.

THEOREM 5.1 Consider the initial value problem for equation (3.1) posed in $Q=\mathbb{R} \times(0, \infty)$ with power function $\Phi(s)=s^{m} / m$. If $m>-1$ then for every nondecreasing $u_{0}$ with $u_{0}(-\infty)=0$, $u_{0}(\infty)=1$, there exists a unique continuous weak solution in the sense $u(x, t) \geqslant 0$ such that $u(\cdot, t)$ jumps from 0 to 1 as $x$ ranges over the line $x \in \mathbb{R}$. If $m \leqslant 0$ the last condition is essential to ensure uniqueness. This class of solutions has bounded gradients for strictly positive times $(t \geqslant \tau>0)$, and spreads in space as time advances and the asymptotic decay formula 5.1 holds for $v=u_{x}$.

On the contrary, if $m \leqslant-1$ solutions for this initial value problem with bounded data do not exist.

The results of the power case reflected in this theorem can be generalized to equations with more general $\Phi$. Only the behaviour of $\Phi(s)$ at $s=0$ and $s=\infty$ will determine the different behaviour types. In order to tackle the first, we assume that the initial data (and hence the solutions) $v$ are bounded. Then it is known that the condition for existence with finite mass is

$$
\int_{0}^{s} \Phi^{\prime}(s) s \mathrm{~d} s<\infty .
$$

The question of large arguments is similar to the power case. These issues will be discussed at length in [57]. For a study of these questions in the class of self-similar solutions cf. [30].

\subsection{Existence of sharp interfaces}

There is an interesting subclass of equations (3.1), or (4.1), where sharp interfaces appear. Let us look first at power nonlinearities. Indeed, for exponent $m>1$ solutions of the Cauchy problem for equation (3.1) with initial data having compact support will keep this property for all times, while infinite propagation occurs whenever $m \leqslant 1$ (cf. [13, 36]). In the first case, given an integrable function $u_{0} \geqslant 0$ with integral 1 the solution of the problem can be seen as a classical solution of equation 3.1 in a domain

$$
\Omega=\{(x, t):-l(t)<x<r(t)\},
$$


with initial conditions $u(x, 0)=u_{0}(x)$ and boundary conditions

$$
\left\{\begin{array}{lll}
u(x, t)=0, & \Phi\left(u_{x}\right)=0 & \text { for } x=l(t), \\
u(x, t)=1, & \Phi\left(u_{x}\right)=0 & \text { for } x=r(t) .
\end{array}\right.
$$

The lines $x=l(t)$ and $x=r(t)$ are called interfaces or moving boundaries and are completely determined by the above-specified conditions. They are known to be smooth (analytic) functions of $t$ [4, 8], and diverge as $t \rightarrow \infty$ like $O\left(t^{\gamma}\right)$, thus giving a quantitative estimate of the dispersion effect. We remark in passing that the presence of interfaces means also that the equation is not uniformly parabolic at those points, and consequently the solutions have limited regularity.

On the other hand, when $m \leqslant 1$ the same Cauchy problem leads to positive solutions with $l(t)=-\infty$ and $r(t)=\infty$. The property of null flux is equivalent to imposing $u_{x}=0$ at $\pm \infty$, a quite natural condition in view of the values $u=0,1$ at $\pm \infty$. This condition is automatic for $m>0$. However, for $m \leqslant 0$ we can have new solutions with decreasing total mass, i.e., such that

$$
\frac{\mathrm{d}}{\mathrm{d} t} \int v(x, t) \mathrm{d} x<0
$$

and they can be even forced to vanish identically in finite time by controlling the outgoing flux at $x= \pm \infty$. We refer to [45, 46] for a detailed analysis. In the integrated version they would lead to solutions with a restricted grey range. For the more general class of $\Phi$ mentioned above the condition to have finite interfaces is

$$
\int_{0}^{s} \frac{\Phi^{\prime}(s)}{s} \mathrm{~d} s \leqslant \infty
$$

The fact that this condition is necessary and sufficient can be easily seen on the family of travelling waves. The property was first pointed out in [41].

\section{Solutions of Type II. Conjugate formulations}

We now address the main question of this paper, the construction of solutions with large gradients, appropriate for the contour enhancement problem.

\subsection{Basic Free Boundary Problem}

It is formulated as follows:

Given an increasing function $u_{0}(x)$ defined in an interval $(a, b)$ with end values $u(a+)=0$, $u(b-)=1$, find a continuous function $u(x, t)$ and continuous curves $x=l(t)$ and $x=r(t)$ such that

(i) $l(0)=a, r(0)=b$, and $l(t)<r(t)$ for some time interval $t \in(0, T)$,

(ii) $u$ is $C_{x, t}^{2,1}$ and solves the following problem in $\Omega=\{(x, t): 0<t<T, l(t)<x<r(t)\}$ :

$$
\left(\mathrm{P}_{\mathrm{II}}\right) \quad \begin{cases}u_{t}=\Phi\left(u_{x}\right)_{x} & \text { in } \Omega, \\ u(x, 0)=u_{0}(x) & \text { for } a \leqslant x \leqslant b, \\ u(l(t), t)=0, u_{x}(l(t), t)=+\infty & \text { for } 0<t<T, \\ u(r(t), t)=1, u_{x}(r(t), t)=+\infty & \text { for } 0<t<T .\end{cases}
$$


Such a triple $(u, l, r)$ is called a solution of Type II. The regularity required from $u$ as a solution of the problem will depend on the generality of the data. At least $u$ will be continuous in the closure of $\Omega$. Furthermore, to avoid unnecessary generality we will require $u$ to be smooth in the interior of $\Omega$. Finally, the requirement of monotonicity is not intrinsic from the mathematical point of view, but it suits the application and allows for the use of the powerful conjugate formulations. Here is the existence result that we are going to prove:

THEOREM 6.1 Let $\Phi$ be a flux function that is defined, smooth and with $\Phi^{\prime}(s)>0$ for all $s>0$. Assume moreover that $\Phi(\infty)$ is finite. Then for every increasing function $u_{0}(x)$ defined in an interval $[a, b]$ with $u(a)=0, u(b)=1$ and $u_{0}^{\prime} \geqslant c>0$ there exists a unique continuous function $u(x, t)$ which is defined in a set $\Omega$ as above, is smooth and strictly monotone in $x$ for $0<u<1$, and there exist continuous curves $l(t)$ and $r(t)$ such that the triple $(u, l, r)$ solves problem $\left(\mathrm{P}_{\mathrm{II}}\right)$ in $\Omega_{T}$. Moreover, $u_{x} \geqslant c>0$ whenever $0<u<1$.

We will also show in our construction that the boundary curves are monotone: $l(t)$ is nondecreasing, $r(t)$ nonincreasing. On the other hand, there is the problem of determining whether $T$ is finite or infinite. This depends on $\Phi$ as we will see below.

\subsection{Conjugate formulation}

When dealing with smooth monotone solutions $u_{x}>0$ we can invert the variables $x$ and $u$ and write $x=X(u, t)$. Then $u_{x} \cdot x_{u}=1$, and after some computations we get the partial differential equation satisfied by $x$ as a function of $u$ and $t$ :

$$
x_{t}=\left(\Psi\left(x_{u}\right)\right)_{u},
$$

where $\Psi$ is the conjugate flux function (conjugate to $\Phi$ ), defined for $s>0$ as

$$
\Psi(s)=-\Phi(1 / s) .
$$

Differentiation of equation 6.1 with respect to $u$ gives rise to the differentiated conjugate equation for $w=\partial x / \partial u$ as a function of $u$ and $t$ :

$$
w_{t}=(\Psi(w))_{u u} .
$$

We complete the list of related equations with the direct differentiated equation for $v=\partial u / \partial x$, already seen:

$$
v_{t}=(\Phi(v))_{x x},
$$

and then $v=1 / w$. It is important to point out that these relations are equivalent to the well known Bäcklund transform [23] between the main variables $v$ and $w$ of the second and fourth formulations. Indeed, we have

$$
v(x, t)=\frac{1}{w(u, t)}, \quad u(x, t)=c+\int_{\Gamma}\left(v \mathrm{~d} x+\Phi(v)_{x} \mathrm{~d} t\right) .
$$

The reader is referred to [46, 55, 56] for other applications of this technique.

\subsection{Posing and solving the conjugate problems}

We now show how to use the conjugate formulations to solve the original problem. We assume that $\Phi$ is a flux function defined for all $s>0$ and such that $\Phi(\infty)$ is finite, say, $\Phi(\infty)=0$. Then $\Psi(s)$ 
is continuous at $s=0$ and $\Psi(0)=0$. This is the class of flux functions for which the conjugate problem looks simpler. Since we have assumed that $\Phi$ is smooth, so is $\Psi$ in its domain of definition.

(i) Since we are interested in solving the conjugate problem as an auxiliary step for Problem $\left(\mathrm{P}_{\mathrm{II}}\right)$ we will relate the initial values for the function $w(u, t)$ to $u_{0}$ as follows. Assuming that $u_{0}$ is continuous and strictly monotone in the interval $I=\{a<x<b\}$, with $u_{0}(a)=0, u_{0}(b)=1$, and $C^{1}$ smooth inside $I$ with $\mathrm{d} u_{0} / \mathrm{d} x$ bounded below away from zero, we define the inverse function $x=h(u)=$ $u_{0}^{-1}(u):[0,1] \rightarrow[a, b]$. Then we set

$$
w_{0}(u)=\frac{1}{u_{0, x}(h(u))},
$$

which is defined for $0 \leqslant u \leqslant 1$ and is positive, bounded and smooth inside, i.e., for $u \in(0,1)$.

(ii) We then solve the conjugate problem

$$
\left(\mathrm{P}_{\mathrm{c}}\right) \quad \begin{cases}w_{t}=\Psi(w)_{u u} & \text { for } 0<u<1, t>0, \\ w(u, 0)=w_{0}(u) & \text { for } 0 \leqslant u \leqslant 1, \\ w(u, t)=0 & \text { for } u=0,1 .\end{cases}
$$

As initial data we choose a nonnegative, bounded function $w_{0}$. Under these conditions Problem $\left(\mathrm{P}_{c}\right)$ has a unique solution by virtue of well known nonlinear parabolic theory described in the previous section (cf. [14, 16, 17]); but note that now we are dealing with the homogeneous Dirichlet problem. The solution can be obtained as limit of the solutions $w_{\varepsilon}(u, t) \geqslant \varepsilon$ of the nondegenerate problems with initial data $w_{0, \varepsilon}(u)=w_{0}(u)+\varepsilon, \varepsilon>0$. In the monotone limit we get

$$
\lim _{\varepsilon \rightarrow 0} w_{\varepsilon}(u, t)=w(x, t),
$$

which is nonnegative, continuous and bounded. Under the additional assumption that $w_{0}$ is locally bounded away from zero, it is easily proved that the solution $w(u, t)$ is positive, hence classical, in a strip

$$
S_{T}=\{(u, t): 0<u<1,0<t<T\} .
$$

(iii) We also need to know something about the large time behaviour of the solutions to this problem. In the full generality ( $\Psi$ continuous at $s=0$ ), it can be proved rather easily that $w(x, t)$ goes to zero in uniform norm as $t \rightarrow \infty$. However, the rate depends on $\Psi$, as we will see in detail in the next sections. Indeed, depending on $\Psi$ it may happen that the solution vanishes identically after a finite time $T>0$ (so-called finite-time extinction). For power nonlinearities $\Psi^{\prime}(s)=s^{q-1}$ this happens iff $0<q<1$ (see [18]). On the other hand, it is well known that for $q \geqslant 1$ the decay rate is $O\left(t^{-1 /(q-1)}\right)$, while for $q=1$ it is exponential.

(iv) Next, we pass to the integrated version using the formula

$$
z(u, t)=\int_{\Gamma}\left(w \mathrm{~d} u+\Psi(w)_{u} \mathrm{~d} t\right),
$$

where $\Gamma$ is any piecewise smooth curve in $(u, t)$ space starting from a fixed point, say $u=1 / 2$, $t=0$, and arriving at a generic point $(u, t)$. In this way we obtain a solution of the integrated equation $z_{t}=\left(\Phi\left(z_{u}\right)\right)_{u}$, much as we did in the case of the original pair of formulations. Note that

$$
z(1, t)-z(0, t)=\int w \mathrm{~d} u \rightarrow 0
$$

as $t \rightarrow T$. 
(v) Let us examine the curves $z_{(u)}(t)=z(u, t)$ for fixed $u \in[0,1]$. It is clear from the smoothness of the solutions that these curves are $C^{\infty}$ smooth for every $0<u<1$. We are interested in the limit curves

$$
l(t)=\lim _{u \rightarrow 0} z(u, t), \quad r(t)=\lim _{u \rightarrow 1} z(u, t) .
$$

The limit is well defined for every $0<t<T$ by monotonicity of $z$ as a function of $u$. These curves will show up in the next subsection as the interfaces of the original problem.

LEMMA 6.2 The curves $z=l(t)$ and $z=r(t)$ are continuous and monotone for all $0<t<T$ with $a \leqslant l(t)<r(t) \leqslant b(r(t)$ is nonincreasing and $l(t)$ nonincreasing). As $t \rightarrow T$ we have $r(t)-l(t) \rightarrow 0$.

Proof. For $0<s<t<T$ and $0<u_{1}<u_{2}<1$ we have

$$
z\left(u_{1}, t\right)-z\left(u_{1}, s\right)=-\int_{u_{1}}^{u_{2}} w(u, t) \mathrm{d} u+\int_{u_{1}}^{u_{2}} w(u, s) \mathrm{d} u+\left(z\left(u_{2}, t\right)-z\left(u_{2}, s\right)\right) .
$$

Fix now $s>0$ and let $t$ be a bit larger than $s$. Since $w$ is bounded uniformly for $t \geqslant s$ the two integrals are uniformly small as long as $u_{1}-u_{2}$ is small. We will fix now $u_{2} \sim 0$ and let $u_{1} \in\left(0, u_{2}\right)$ go to 0 . It is clear then that $z\left(u_{1}, t\right)-z\left(u_{1}, s\right)$ is uniformly small and goes to 0 as $t \rightarrow s$. Hence, $l(t)$ is continuous at $t=s$. The argument for $r(t)$ as $u \rightarrow 1$ is the same.

For classical solutions the monotonicity of the limit curves is a consequence of the equation

$$
\frac{\partial z}{\partial t}=\frac{\partial}{\partial u} \Phi(w)
$$

which must be positive at $u=0$ and negative at $u=1$ because $w \geqslant 0$ and we have zero boundary conditions. For the general case we use the dependence of the solutions on $\Phi$ as demonstrated in [16]. Hence, $l^{\prime}(t) \geqslant 0, r^{\prime}(t) \leqslant 0$. We will see another proof below. Note that the rest of the curves $z_{(u)}(t)$ need not be monotone (unless $w_{0}$ is a rearranged symmetrical function for instance).

As for the last statement, it follows immediately from 6.7 It will mean for the original problem that the solution concentrates into a vertical profile, thus showing the formation of the desired front.

\subsection{Inversion. Solutions of Type II}

Thanks to the fact that $\partial z / \partial u=w>0$, we can invert the dependence between $z$ and $u$ in the previous construction to get a function $u=u(z, t)$ that is easily shown to satisfy the equation

$$
u_{t}=\left(\Phi\left(u_{z}\right)\right)_{z}
$$

Moreover, $u$ is a monotone function of $z$ and takes the values $u=0$ and $u=1$ respectively at the left and right endpoints of the domain of definition

$$
\Omega_{z}=\{l(t)<z<r(t)\}, \quad l(t)=z(0, t), \quad r(t)=z(1, t) .
$$

where $z(\cdot, t)$ is the function defined in (6.6). Therefore, $u(z, t)$ is a candidate to solve our original problem if we identify the independent variable $z$ with $x-c$, where $c$ is uniquely determined by the relation $u_{0}(c)=1 / 2$. 
In order to check that we have solved the original problem we still have to check some particulars. It is clear that $v=u_{x}$ is related to the original $w$ by the formula

$$
v(x, t)=\frac{1}{w(u, t)},
$$

which simply states the derivative rule for the inverse function, and $u$ in the second member is given by $u(z, t), z=x-c$, as explained before. Here comes an important point: since $w$ takes on zero boundary values, $v(x, t)$ diverges at the endpoints of its domain of definition, $\Omega$. In other words, the solutions of the original problem $u=u(x, t)$ enjoy the property of infinite gradients at the endpoints of the strip where they are defined. Since $\Phi(\infty)=0$ this also means zero flux at these points, a reasonable requirement, which explains why this condition has to be imposed on $\Phi$.

As for the initial data, we have the mass formula

$$
x=\int_{1 / 2}^{u} w_{0}(u) \mathrm{d} u+c \quad \text { with } u_{0}(c)=\frac{1}{2},
$$

so that $x$ ranges over an interval $[a, b]$ when $u$ goes from 0 to 1 , i.e., $a=z(0,0)+c, b=z(1,0)+c$. This rule is accompanied by the rule $u=\int_{l(t)}^{x} v(x, t) \mathrm{d} x$.

\subsection{Uniqueness}

Uniqueness of our class of monotone solutions works by translating any couple of solutions of Problem $\left(\mathrm{P}_{\mathrm{II}}\right)$ with the same initial data into the conjugate formulation. They continue to have the same initial data. Uniqueness of weak solutions is well known for that equation. The Theorem is proved.

\subsection{Front formation}

The asymptotic formation of a vertical front is a simple consequence of the fact that there exists the limit

$$
\lim _{t \rightarrow T} r(t)=\lim _{t \rightarrow T} l(t)=x_{\infty} \in(a, b) .
$$

The existence of the common limit follows from Lemma 6.2

\section{Uniqueness and comparison for Type II solutions}

The previous construction provides existence and uniqueness of monotone solutions. We tackle next the property of comparison, which is stronger that uniqueness.

ThEOREM 7.1 Comparison applies to the solutions of Theorem 6.1 let $u_{1}(x, t), i=1,2$, be two solutions defined in a strip $S_{T}=\mathbb{R} \times(0, T)$ having initial data $u_{0 i}$, where the solutions and the data have been extended by 0 to the left of the definition domain, by 1 to the right. If $u_{01}(x) \geqslant u_{02}(x)$, then for all $x \in \mathbb{R}$ we have

$$
u_{1}(x, t) \geqslant u_{2}(x, t) \quad \text { in } S_{T} .
$$

Proof. The main idea is to use a contradiction argument at touching points of ordered solutions. We proceed in several stages and need a definition, taken from the theory of viscosity solutions in [25]. We say that two solutions are strictly separated at a time $t$ if $u_{1}>u_{2}$ at all points intermediate to the bounding interfaces, and these are also separated. 
(1) Given two solutions $u_{1}$ and $u_{2}$ with ordered data we must obtain approximations to which the contradiction argument can be applied. First, we separate the initial data of the solutions by displacing the first solution $u_{1}$ to the left (the problem is invariant under space displacements). Next, assume that we have modified $u_{1}$ so that it has a finite slope on the left-hand free boundary, and $u_{2}$ so that it has a finite slope on the right-hand free boundary. In this situation we argue on the first point and time $\left(x_{0}, t_{0}\right)$ where the graphs of both solutions touch, and discover that: (i) it cannot happen with value $u \in(0,1)$ by the Strong Maximum Principle; (ii) not on the left-hand side where $u_{1}=u_{2}=0$, because at such a point we have the right derivative $u_{2, x}=+\infty$ while $u_{1, x}$ is finite, which contradicts the fact that we must still have $u_{1}\left(\cdot, t_{0}\right) \geqslant u_{2}\left(\cdot, t_{0}\right)$; (iii) same argument applies to the right-hand side where $u_{1}=u_{2}=1$. We conclude that such solutions $u_{1}$ and $u_{2}$ cannot touch, hence strict separation is preserved in time, and comparison $u_{1} \geqslant u_{2}$ holds in the strong sense, i.e., with strict separation at all times.

(2) We must now prove that the original solutions can be approximated by solutions with finite derivatives on the lateral boundaries. This can be obtained by an easy modification of the previous construction. Thus, if $u$ is the constructed solution, it can be approximated at the level of the conjugate problem by putting the value $w=\varepsilon$ on the left-hand boundary $u=0$. It is a rather standard monotonicity argument that $w_{\varepsilon} \rightarrow w$ as $\varepsilon \rightarrow 0$. Undoing the transformation this means that $u_{\varepsilon}$ converges to the original constructed solution $u$. A similar construction applies to $u_{2}$ by modifying $w_{2}$ at the border $u=1$. After displacement of $u_{\varepsilon}$ we get $u_{1, \varepsilon}(x+\delta, t) \geqslant u_{2, \varepsilon}(x, t)$. Let now $\varepsilon \rightarrow 0$.

We next derive another proof of the monotonicity of the interfaces.

COROLLARY 7.2 The interfaces are monotone in all cases.

Proof. Given a solution $u$ with initial interfaces $a$ and $b$, we may place a very steep solution $\hat{u}$ to the left, i.e., with $\hat{u}=0$ for $x \leqslant a-2 \varepsilon, \hat{u}=1$ for $x \geqslant a-\varepsilon$. If $\hat{u}^{\prime}$ is symmetric then the interfaces are monotone, hence the left interface of $\hat{u}$ lies to the right of $a-2 \varepsilon$. Then $\hat{u} \geqslant u$ for all times, hence $l(t) \geqslant a-2 \varepsilon$, and in the limit $l(t) \geqslant a$. The same argument applies by taking the origin of times at any $y \in(0, T)$, hence $l(t)$ is increasing in $(0, T)$. Likewise, $r(t)$ is proved to be decreasing.

\section{Self-similarity and asymptotics for power nonlinearities}

In the power case, where $\Phi(s)=(1 / m) s^{m}$, the asymptotic condition " $\Phi(\infty)$ finite" means $m<0$. Then $\Psi(s)=(1 / q) s^{q}$ with $q=-m>0$. Reminder: we consider only monotone solutions of 3.1. and nonnegative solutions of 4 (4.1), and $q=1+2 \alpha$ in the notation of the first section. Therefore, $\alpha>-1 / 2$ means $q>0$.

There is a unique solution of problem $\left(\mathrm{P}_{\mathrm{c}}\right)$ with initial data as in Section 6 The large time behaviour depends on the exponent. For $q \geqslant 1$ solutions are defined and nontrivial globally in time, while for $0<q<1$ they exist only in a finite interval $0<t<T$ and $w(x, t) \rightarrow 0$ uniformly as $t \rightarrow T$.

As for the exact behaviour, if $\alpha \neq 0$ the self-similar behaviour reduces to writing $U=\Phi(\xi)$ with $\xi=x t^{1 / 2 \alpha}$ and integrating the ODE

$$
\Phi^{\prime \prime}=\frac{1}{2 \alpha} \xi\left(\Phi^{\prime}\right)^{2+q}
$$


to obtain in the monotone region

$$
\Phi^{\prime}(\xi)=A\left(K^{2}-\xi^{2}\right)^{-1 /(2+2 \alpha)}, \quad A=(2 \alpha /(1+\alpha))^{1 /(2+2 \alpha)},
$$

and a constant $K>0$ determined by the end values $\min \Phi(\xi)=0, \max \Phi(\xi)=1$. For the largetime behaviour of general solutions we have to make a study in different cases. We start with $\alpha=0$ which was not included before.

Case $\alpha=0$, hence $q=1$. The conjugate equation is the linear heat equation. It is well known that the solutions behave for large times like

$$
w(u, t) \sim C_{1} W(u, t) \equiv C_{1} e^{-\lambda t} f_{1}(u),
$$

where $\lambda=\pi^{2}$ is the first eigenvalue of the Laplacian in $[0,1], f_{1}(x)=\sin (\pi u)$ is the first eigenfunction, and $C_{1}>0$ is a constant that depends on the initial data. Here and in what follows we denote by capital letters the quantities corresponding to self-similar solutions. We get the intermediate step

$$
x-c=z(u, t) \sim Z(u, t) \equiv-C e^{-\lambda t} \cos (\pi u),
$$

with $C=C_{1} / \pi$. This formula can be viewed as an implicit expression of $u=u(x, t)$ defined in the space between the interfaces. Note that $u=1 / 2$ for $x=c$ and all $t>0$. Putting $c=0$ we get for the self-similar solution

$$
U(x, t)=F\left(x e^{\pi^{2} t} / C\right),
$$

where $F^{\prime}$ behaves like $d^{-1 / 2}$ near the end values or interfaces, $d$ being the distance to these points. Moreover, the interfaces are

$$
R(t)=C R_{1}(t), \quad L(t)=-C R_{1}(t), \quad \text { with } R_{1}(t)=e^{-\pi^{2} t} .
$$

These estimates become the first order approximation when we consider general solutions:

$$
u(x, t) \sim F\left(x e^{-\pi^{2} t} / C\right), \quad r(t), l(t) \sim c \pm C R_{1}(t) .
$$

The gradients are given by

$$
u_{x}(x, t)=\frac{1}{w} \sim \frac{1}{C} e^{\lambda t} \frac{1}{\sin (\pi u)} .
$$

They blow up exponentially as $t \rightarrow \infty$ and like $O\left(d^{-1 / 2}\right)$ at the interfaces, where $d$ is the distance to the interface.

Case $\alpha>0$, hence $q>1$. This is the case treated in [10] by the direct self-similar method. We recover the behaviour of general solutions from the conjugate problem. We have a self-similar asymptotic expression in the form of separation of variables:

$$
w(u, t) \sim W(u, t) \equiv t^{-1 /(q-1)} f_{q}(u),
$$

where $f_{q}(u)>0$ is the unique solution of the associated elliptic problem

$$
\left(f^{q}\right)^{\prime \prime}+\mu f=0 \quad \text { in }[0,1], \quad \mu=\frac{q}{q-1},
$$


and $f=0$ at the endpoints. This problem was studied in [7]. In this case there is no free constant, i.e., the behaviour is universal. From it we get the intermediate asymptotic estimates

$$
x-c=z(u, t) \sim t^{-1 /(q-1)} g_{q}(u) .
$$

This formula defines implicitly $u=u(x, t)$ in the space between the interfaces as

$$
u \sim F_{q}\left((x-c) t^{1 /(q-1)}\right) .
$$

Since $f(u)$ behaves exactly like $O\left(u^{1 / q}\right)$ near the endpoint $u=0$, and $O\left((1-u)^{1 / q}\right)$ near $u=1$, it follows after integration and inversion that $u=F$ behaves like $O\left(d^{(q /(q+1)}\right)$, where $d$ is the distance to the interfaces. These are given by

$$
l(t)=c-C_{q} t^{-1 /(q-1)}, \quad r(t)=c+C_{q} t^{-1 /(q-1)} .
$$

The gradient is given by

$$
u_{x}(x, t) \sim t^{1 /(q-1)} \frac{1}{f_{q}(u)},
$$

and blows up like $O\left(t^{1 / 2 \alpha}\right)$ as $t \rightarrow \infty$ and like $O\left(d^{-\gamma}\right)$ at the interfaces, where $\gamma=1 /(q+1)=$ $1 /(2 \alpha+2)$.

Case $-1 / 2<\alpha<0$, hence $0<q<1$. This is a new case, not included in the modelization of [40, 10]. We get solutions from the conjugate problem which exist for a finite time $T$ and behave as $t \rightarrow T$ like the separation of variables formula:

$$
w(u, t) \sim(T-t)^{1 /(1-q)} f_{q}(u),
$$

where $f_{q}(x)>0$ is the unique solution of the associated elliptic problem

$$
\left(f^{q}\right)^{\prime \prime}+\mu f=0 \quad \text { in }[0,1], \quad \mu=\frac{q}{q-1},
$$

and $f=0$ at the endpoints, with no free constant. We get by integration

$$
x-c=z(u, t) \sim(T-t)^{1 /(1-q)} g_{q}(u) .
$$

This formula defines implicitly $u=u(x, t)$ in the space between the converging interfaces

$$
l(t)=c-C(T-t)^{1 /(1-q)}, \quad r(t)=c+C(T-t)^{1 /(1-q)} .
$$

The gradient is given by

$$
u_{x}(x, t) \sim(T-t)^{-1 /(1-q)} \frac{1}{f_{q}(u)},
$$

and blows up like $O\left((T-t)^{1 / 2 \alpha}\right)$ as $t \rightarrow T$ and like $O\left(d^{-\gamma}\right)$ at the interfaces, $\gamma$ as before. 


\section{Asymptotic estimates for more general $\Phi$}

Assumptions on $\Phi$ to behave like a power at infinity guarantee that the asymptotic behaviour is as predicted in the power case. Thus, Bertsch and Peletier study in [22] the asymptotic behaviour of equation 6.1 written in the form

$$
\beta(v)_{t}=v_{x x} .
$$

In our application the space variable $x$ becomes $u$ and $\beta=\Psi^{-1}$. They assume that $\beta(0)=0$, $\beta^{\prime}(s)>0$ for $s>0$ and

$$
-1 \leqslant \frac{s \beta^{\prime \prime}(s)}{\beta^{\prime}(s)} \leqslant \alpha
$$

for all small $0<s<s_{0}$ and some $\alpha \in(0,1)$. They also need the more stringent condition that the limit

$$
\sigma(s)=\lim _{\varepsilon \rightarrow 0} \frac{s \beta^{\prime \prime}(\varepsilon s)}{\beta^{\prime}(\varepsilon)}
$$

exists for every $s>0$. This limit is necessarily of the form $\sigma(s)=s^{m}$ for some $m \in[0,1)$. Those conditions cover in particular powers $\Psi(s)=s^{q}$ with $q=1 / m>1$, as well as the exponential $\Psi(s)=e^{-1 / s}$, and any other function resembling such examples near $s=0$.

Under these restrictions, they establish an asymptotic separation-of-variables result that, when translated to our setting, means that the solution $w(u, t)$ of the Cauchy problem (6.1) behaves as $t \rightarrow \infty$ in the separate variable form $w \sim y(t) f(u)$, more precisely,

$$
\lim _{t \rightarrow \infty} \frac{\beta(w)}{y(t)}=f(u)
$$

where $y(t)$ is the solution $y^{\prime}=-y^{1 / q}$, i.e., $y(t) \sim c t^{-q /(q-1)}$ and $f$ is the profile obtained as in the previous section. This means that the analysis of the previous section is justified for almost power-like flux functions if they resemble a power with exponent $q>1$. The cases $q=1$ and $q<1$ should be justified in a similar manner. We note that a similar calculation for the Cauchy problem was done in [43].

We point out that these restrictions on the nonlinearity are satisfied by the equation proposed in the image processing model, (1.4), hence the results apply to that model.

\section{Numerical experiments}

We have computed the solutions of the free boundary problem $\left(\mathrm{P}_{\mathrm{II}}\right)$ by solving the conjugate problem, which is a homogeneous Neumann problem, in the cases $\alpha=0, \alpha=1$ and $\alpha=-1 / 2$. The conjugate equations are $p$-Laplacian equations with $p=2,4$ and $3 / 2$. The computations have been done with symmetrical data $x_{0}(u)=-\cos (\pi u)$ and asymmetrical $x_{0}(u)=2 u^{4}-(1 / 2) u^{6}-1$. The last two examples stabilize in finite time (since $\alpha<0)$.

In Figures $1-6$, the horizontal axis is $x$, vertical is $u$, and the curves are parametrized by time, evolving with increasing $t$ towards the sharp front.

\section{Discussion and conclusions}

We have established the well-posedness of a free boundary problem that represents a onedimensional version of the model for image contour enhancement. We have also established the 

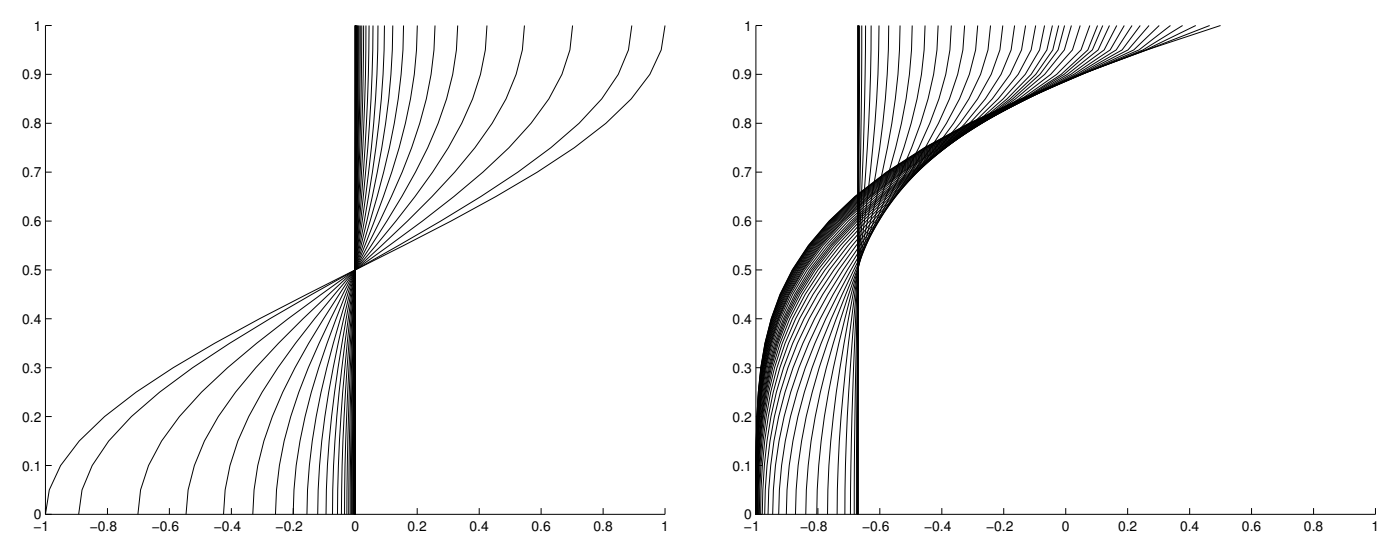

Figures 1 and 2 . The case $\alpha=0$ with symmetrical and asymmetrical data.
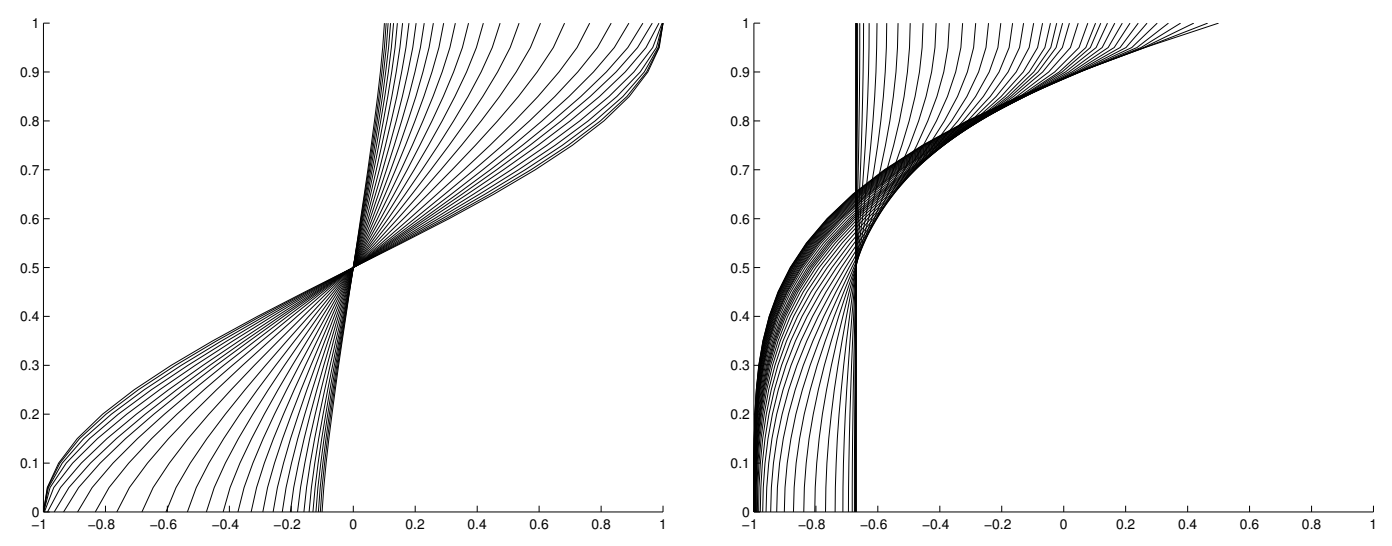

Figures 3 and 4 . The case $\alpha=1$ with symmetrical and asymmetrical data.
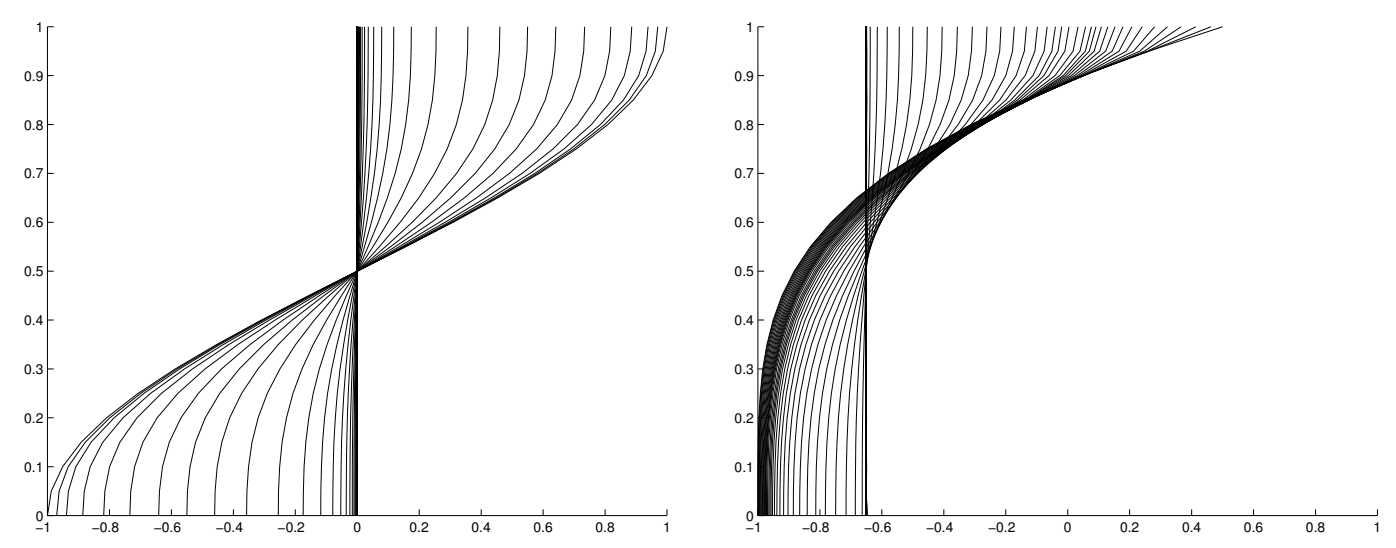

Figures 5 and 6 . The case $\alpha=-1 / 2$ with symmetrical and asymmetrical data. 
asymptotic behaviour and its rates. The results apply to a large class of equations, which enlarges the scope of the results of [10,40]. We have performed a classification of the solutions and their properties according to the properties of the constitutive function $\Phi$.

We have used as a framework the 1D theory of nonlinear parabolic equations of diffusive type, or nonlinear diffusion equations for short. These equations are typically used in describing processes of mass diffusion or thermal propagation (with or without additional effects, like convection or reaction). Here they appear in image processing. The types used here cover the model cases known as $p$-Laplacian equations and porous medium equations or their variants.

The analysis is technically performed by means of a series of remarkable transformations that lead to conjugate problems that are easier to analyze. These transformations are related to the Bäcklund transform.

Note that in our problem setting, the equation is forward parabolic, and the backward movement of the interface is due to the effect of the singular boundary condition, which happens to be compatible with the equation for the appropriate class of functions $\Phi$ (e.g., for powers $m<0$ ).

There are a number of interesting consequences of the mathematical analysis that we sum up next.

- Mathematical generality. The first one is the observation that solutions with gradient blowup can be obtained for the nonlinear diffusion equation with a large class of constitutive functions. Namely, $\Phi$ can be any continuous nondecreasing function defined for $s>0$ such that $\Phi(\infty)$ is bounded. We have made for convenience the assumption of smoothness and strict monotonicity for $0<s<\infty$. Actually, the constitutive function can be more general, even a discontinuous maximal monotone graph as in [14,24]. But such a generality is not practical here, though the classification and results go through after some heavy work. A general presentation of that generality will be the object of the work [57].

On the other hand, the application of similar techniques to the original $2 \mathrm{D}$ model is still a problem under investigation.

- Focusing in finite time. In the case of powers $\Phi(s) \sim s^{m}$ the condition means $m<0$, and not only $m \leqslant-1$ as considered before. Moreover, the new range $0>m>-1$ leads to a very fast evolution which arrives at a vertical front in finite time. This idea might have an applied interest.

- Coexistence of types. We also have a so-called class of solutions of Type I that fall into the scope of the standard parabolic theory. They exist under the condition that the integral $\int\left(\Phi^{\prime}(s) / s\right) d s$ converges at $s=\infty$, which in the case of powers means $m>-1$. This fact leads to an interesting observation: there is a range of co-existence of solutions of Type I and II for $-1<m<0$, i.e., $-1 / 2<\alpha<0$. This may be of interest from the theoretical point of view: two different problems share the same equation and same initial data, but the solutions differ as a consequence of a further choice: of existence or not existence of a free boundary.

- The pressure. There is a way of formally unifying the standard theory of free boundary solutions for the porous medium equation with the present theory of free boundaries in fast diffusion. This works by means of the new variable called pressure that for an equation of the form $v_{t}=\Phi(v)_{x x}$ is defined as

$$
p(v)=\int_{a}^{v} \frac{\Phi^{\prime}(s)}{s} \mathrm{~d} s .
$$

If the integral is convergent at $v=0$ then the choice $a=0$ is made. Otherwise, any value in the domain of $\Phi$ is good. Indeed, the similarity in the behaviour of the pressure of Porous Medium case, 
$\Phi(v)=v^{m}, m>1$, and our case (which lies in the power range $m \leqslant 0$ ) is seen when we write the partial differential equation for $p$ which reads

$$
p_{t}=\sigma(p) \Delta p+|\nabla p|^{2}
$$

with $\sigma(p)=\Phi^{\prime}(v)$. For $\Phi(v)=c v^{m}$ we get $p=c m v^{m-1} /(m-1)$ and $\sigma(p)=(m-1) v$. Note that with this definition $p \leqslant 0$. The paper [55] explores further the properties of free boundary solutions for the pressure of fast diffusion equation. Note that, contrary to the porous medium case where the support of the pressure solutions expands in time, in our case it shrinks in time.

- Grey levels as end values. Another variation of the main theme is to consider solutions losing the white or black level. This is investigated in the paper [46], where many solutions of the differentiated equation for $v$ are obtained in the range $0 \geqslant m>-1$ with the same initial data by assigning fluxes at infinity:

$$
\lim _{x \rightarrow \infty}\left(v^{m-1} v_{x}\right)=-f(t), \quad \lim _{x \rightarrow-\infty}\left(v^{m-1} v_{x}\right)=g(t),
$$

for bounded functions $f, g \geqslant 0$. In terms of $u$ it means in particular that we can obtain a free boundary solution with a decreasing value of highest colour level,

$$
u(\infty, t)=F(t)=1-\int_{0}^{t} f(t) \mathrm{d} t<1
$$

Analogously, we can impose an increasing value for the lowest colour level,

$$
u(-\infty, t)=G(t)=\int_{0}^{t} g(t) \mathrm{d} t>0 .
$$

- Nonmonotone fronts. There is a gap in the theory we have developed, namely that we assumed that the front is monotone in the space direction, in other words, that $u$ is nondecreasing as a function of $x$. The limitations of fast diffusion equations to admit nonmonotone solutions are not accidental. In the paper [47] it is proved that the model equation $u_{t}=u_{x}^{m-1} u_{x x}$ does not admit non-monotone solutions if $m \leqslant 0$. In terms of the equation for $v=u_{x}$ it means that there are no solutions with both signs. However, there is hope when using functions $\Phi$ like in (1.4) that are degenerate at infinity, i.e., $\Phi^{\prime}(\infty)=0$, but regular at all other values, e.g., $\Phi \in C^{1}(\mathbb{R})$. Indeed, the problem with the monotonicity happens because of the singularity at $u_{x}=0$ which forbids maxima or minima, while our main interest is in the free boundary that is governed by the large values, $u_{x} \rightarrow \infty$. This question leads to a quite interesting extension of the theory of singular free boundary problems that is investigated separately in [2] in the $1 \mathrm{D}$ case. In this work the relation with more classical elliptic parabolic free boundary problems, like those of [21,34,35], is investigated.

- Nonmonotone nonlinearities. In the spirit of the Perona-Malik model, situations can be considered where $\Phi$ is not monotone, hence the equation is not parabolic. A regularization is then needed so that one faces a regularized forward-backward diffusion problem. See in this respect [12] and [60]. This subject is again under study.

Acknowledgments. The work of the first author was supported by Applied Mathematics subprogram of the US Department of Energy under Contract DE-AC03-76-SF00098. The second author has been partly supported by TMR Projects RTN2-2001-00349 and FMRX-CT98-0201, and Proyecto MCYT BFM2002-04572-C02-02. He is also grateful to the Lawrence Berkeley National Laboratory for supporting a research visit. The authors express their thanks to Prof. A. J. Chorin for his stimulating interest in our work. Computations were performed by Raúl Ferreira using Matlab. 


\section{REFERENCES}

1. Alvarez, L., Lions, P.-L., \& Morel, J.-M. Image selective smoothing and edge detection by nonlinear diffusion. II. SIAM J. Numer. Anal. 29 (1992), 845-866. Zbl 0766.65117 MR 93a:35072

2. Amadori, A. L. \& VÁzquez, J. L. Singular free boundary problem from image processing. Preprint, UAM (2003).

3. Andreu, F., Ballester, C., Caselles, V., \& Mazón, J. M. Minimizing total variation flow. Differential Integral Equations 14 (2000), 321-360. Zbl 1020.35037 MR 2002e:35109

4. Angenent, S. B. Local existence and regularity for a class of degenerate parabolic equations. Math. Ann. 280 (1988), 465-482. Zbl 0619.35114 MR 89e:35072

5. AngEnEnT, S. B. Large time asymptotics for the porous media equation. Nonlinear Diffusion Equations and their Equilibrium States, I (Berkeley, CA, 1986), Math. Sci. Res. Inst. Publ. 12, Springer, New York (1988), 21-34. Zbl 0669.35048 MR 90k:35028

6. Aronson, D. G. The porous medium equation. Nonlinear Diffusion Problems, Lectures Notes in Math. 1224, Springer, New York (1986), 1-46. Zbl 0626.76097 MR 88a:35130

7. Aronson, D. G. \& Peletier, L. A. Large time behaviour of solutions of the porous medium equation in bounded domains. J. Differential Equations 39 (1981), 378-412. Zbl 0475.35059 MR 82g:35047

8. Aronson, D. G. \& VÁzquez, J. L. Eventual $C^{\infty}$-regularity and concavity of flows in onedimensional porous media. Arch. Rat. Mech. Anal. 99 (1987), 329-348 Zbl 0642.76108 MR 89d:35081

9. Barenblatt, G. I. Scaling, Self-Similarity, and Intermediate Asymptotics, Cambridge Texts Appl. Math. 14, Cambridge Univ. Press, Cambridge (1996). Zbl 0907.76002 MR 98a:00005

10. Barenblatt, G. I. Self-similar intermediate asymptotics for nonlinear degenerate parabolic freeboundary problems that occur in image processing. Proc. Natl. Acad. Sci. USA 98 (2001), 12878-12881. Zbl 0999.35113 MR 2002i:35212

11. Barenblatt, G. I., Bertsch, M., Chertock, A. E., \& Prostokishin, V. M. Self-similar asymptotics for a degenerate parabolic filtration-absorption equation. Proc. Natl. Acad. Sci. USA 97 (2000), 9844-9848. Zbl 0966.35069 MR 2001d:76115

12. Barenblatt, G. I., Bertsch, M., Dal Passo, R., \& Ughi, M. A degenerate pseudoparabolic regularization of a nonlinear forward-backward heat equation arising in the theory of heat and mass exchange in stably stratified turbulent shear flow. SIAM J. Math. Anal. 24 (1993), 1414-1439. Zbl 0790.35054 MR 94h:35129

13. Barenblatt, G. I. \& Vishik, M. I. On finite velocity of propagation in problems of non-stationary filtration of a liquid or gas. Prikl. Mat. Mekh. 20 (1956), 411-417 (in Russian). MR 18,256a

14. BÉnilan, P. Équations d'évolution dans un espace de Banach quelconque et applications. Thesis, Univ. Orsay (1972).

15. BÉnilan, P. Opérateurs accrétifs et semi-groupes dans les espaces $L^{p}(1 \leqslant p \leqslant \infty)$. Functional Analysis and Numerical Analysis, Japan-France Seminar 1976, H. Fujita (ed.), Japan Soc. Prom. Sci. (1978), 15-53.

16. BÉnilan, P. \& Crandall, M. C. The continuous dependence on $\varphi$ of solutions of $u_{t}-\Delta \varphi(u)=0$. Indiana Univ. Math. J. 30 (1981), 161-177. Zbl 0482.35012 MR 83d:35071

17. BÉnilan, P. \& GARIEPY, R. Strong solutions in $L^{1}$ of degenerate parabolic equations. J. Differential Equations 119 (1995), 473-502. Zbl 0828.35050 MR 96c:35078

18. Berryman, J. G. \& Holland, C. J. Stability of the separable solution for fast diffusion. Arch. Rat. Mech. Anal. 74 (1980), 379-388. Zbl 0458.35046 MR 81m:35065

19. Bertsch, M. \& DAL PASSO, R. Hyperbolic phenomena in a strongly degenerate parabolic equation. Arch. Rat. Mech. Anal. 117 (1992), 349-387. Zbl 0785.35056 MR 93g:35078

20. Bertsch, M., Dal Passo, R., \& UGHi, M. Nonuniqueness of solutions of a degenerate parabolic equation. Ann. Mat. Pura Appl. 161 (1992), 57-81. Zbl 0796.35083 MR 93f:35124 
21. Bertsch, M. \& Hulshof, J. Regularity results for an elliptic-parabolic free boundary problem. Trans. Amer. Math. Soc. 297 (1986), 337-350. Zbl 0643.35051 MR 88c:35157

22. Bertsch, M. \& Peletier, L. A. The asymptotic profile of solutions of degenerate diffusion equations. Arch. Rat. Mech. Anal. 91 (1985), 207-229. Zbl 0597.35065 MR 87c:35089

23. Bluman, G. \& Kumei, S. On the remarkable nonlinear diffusion equation $(\partial / \partial x)[a(u+$ $\left.b)^{-2}(\partial u / \partial x)\right]-(\partial u / \partial t)=0 . J$. Math. Phys. $21(1980), 1019-1023$. Zbl 0448.35027 MR 81g:35043

24. BREZIS, H. Opérateurs maximaux monotones et semigroupes de contractions dans les espaces de Hilbert. North-Holland Math. Stud. 5, North-Holland, Amsterdam (1973). Zbl 0252.47055 MR $50 \# 1060$

25. CAfFARElli, L. A. \& VÁzQUez, J. L. Viscosity solutions for the porous medium equation. Differential Equations (Florence, 1996), M. Giaquinta et al. (eds.), Proc. Sympos. Pure Math. 65, Amer. Math. Soc., Providence, RI (1999), 13-26. Zbl 0929.35072 MR 99m:35029

26. Caselles, V., Morel, J. M., \& SBert, C. An axiomatic approach to image interpolation. IEEE Trans. Image Process. 7 (1998), 376-386. Zbl 0993.94504 MR 2000d:94001

27. Chasseigne, E. \& VÁzquez, J. L. The pressure equation in the fast diffusion range. Rev. Mat. Iberoamericana, to appear.

28. DiBenedetto, E. Degenerate Parabolic Equations, Universitext, Springer (1994). Zbl 0794.35090 MR 94h:35130

29. Esteban, J. R., Rodríguez, A., \& VÁzquez, J. L. A nonlinear heat equation with singular diffusivity. Comm. Partial Differential Equations 13 (1988), 985-1039. Zbl 0686.35066 MR 89h:35167

30. Ferreira, R. \& VÁzquez, J. L. Study of self-similarity for the fast diffusion equation. Adv. Differential Equations 8 (2003), 1125-1152.

31. Friedman, A. \& KAMIN, S. The asymptotic behavior of gas in an $N$-dimensional porous medium. Trans. Amer. Math. Soc. 262 (1980), 551-563. Zbl 0447.76076 MR 81j:35054

32. Gilding, B. H. \& Peletier, L. A. On a class of similarity solutions of the porous media equation. J. Math. Anal. Appl. 55 (1976), 351-364. Zbl 0356.35049 MR 55 \#9691a

33. Guichard, F. \& Morel, J.-M. A note on two classical shock filters and their asymptotics. Scale-Space and Morphology in Computer Vision (Vancouver, 2001), Lecture Notes in Comput. Sci. 2106, Springer, Berlin (2001), 75-84. Zbl 0996.68224

34. Hulshof, J. An elliptic-parabolic free boundary problem: continuity of the interface. Proc. Roy. Soc. Edinburgh Sect. A 106 (1987), 327-339. Zbl 0654.35051 MR 89i:35133

35. Hulshof, J. \& Peletier, L. A. An elliptic-parabolic free boundary problem. Nonlinear Anal. 10 (1986), 1327-1346. Zbl 0624.35081 MR 88b:35211

36. Kalashnikov, A. S. Some problems of the qualitative theory of non-linear degenerate second-order parabolic equations. Russian Math. Surveys 42 (1987), 169-222. Zbl 0642.35047

37. Kichenassamy, S. The Perona-Malik paradox, SIAM J. Appl. Math. 57 (1997), 1328-1342. Zbl 0887.35071 MR 98i:35088

38. KIng, J. R. Self-similar behaviour for the equation of fast nonlinear diffusion. Philos. Trans. Roy. Soc. London A 343 (1993), 337-375. Zbl 0797.35097

39. KRIM, H. \& BAO, Y. Nonlinear diffusion: a probablilistic approach. ICP99, Kobe, Japan.

40. MALlADi, R. \& SETHIAN, J. A. Image processing: flows under min/max curvature and mean curvature. Graphical Models Image Process. 58 (1996), 127-141.

41. OleǏnik, O., Kalashnikov, A. S., \& Chou, Y.-L. The Cauchy problem and boundary-value problems for equations of the type of unsteady filtration. Izv. Akad. Nauk SSSR Ser. Mat. 22 (1958), 667704 (in Russian).

42. OSHER, S. \& RUdin, L. I. Feature-oriented image enhancement using shock filters. SIAM J. Numer. Anal. 27 (1990), 919-940. Zbl 0714.65096 
43. De PABlo, A. \& VÁzquez, J. L. Regularity of solutions and interfaces of a generalized porous medium equation. Ann. Mat. Pura Appl. 158 (1991), 51-74. Zbl 0757.35009 MR 92g:35091

44. Perona, P. \& Malik, J. Scale space and edge detection using anisotropic diffusion. IEEE Trans. Pattern Anal. Machine Intelligence 12 (1990), 629-639.

45. Rodríguez, A. \& VÁzquez, J. L. A well-posed problem in singular Fickian diffusion. Arch. Rat. Mech. Anal. 110 (1990), 141-163. Zbl 0695.76043 MR 91g:35139

46. Rodríguez, A. \& VÁzquez, J. L. Non-uniqueness of solutions of nonlinear heat equations of fast diffusion type. Ann. Inst. H. Poincaré Anal. Non Linéaire 12 (1995), 173-200. Zbl 0837.35077 MR 96d:35061

47. Rodríguez, A. \& VÁzQuez, J. L. Obstructions to existence in fast-diffusion equations. J. Differential Equations 184 (2002), 348-385. Zbl 1007.35042 MR 2003m:35128

48. RUdin, L. I., OSher, S., \& FATEMI, E. Nonlinear total variation based noise removal algorithms. Phys. D 60 (1992), 259-268. Zbl 0780.49028

49. Samarskĭ, A. A., Galaktionov, V. A., Kurdyumov, S. P., \& Mikhăllov, A. P. Blow-up in Problems for Quasilinear Parabolic Equations. Nauka, Moscow (1987) (in Russian). English transl.: Walter de Gruyter, Berlin (1995). Zbl 1020.35001 MR 96b:35003

50. Sochen, N., Kimmel, R., \& Malladi, R. From high energy physics to low level vision. Preprint LBNL-39243, UC-405, E. O. Lawrence Berkeley National Laboratory (1996), 38 pp.

51. Sochen, N., Kimmel, R., \& Malladi, R. A general framework for low level vision. IEEE Trans. Image Process. 7 (1998), 310-318. Zbl 0973.94502 MR 99k:94016

52. VÁzquez, J. L. An introduction to the mathematical theory of the porous medium equation. Shape Optimization and Free Boundaries (Montréal, PQ, 1990), NATO Adv. Sci. Inst. Ser. C Math. Phys. Sci. 380, Kluwer, Dordrecht (1992), 347-389. MR 95b:35101

53. VÁzQUeZ, J. L. Asymptotic behaviour for the PME in the whole space, J. Evolution Equations 3 (2003), 67-118.

54. VÁzQuez, J. L. Asymptotic behaviour for the PME in a bounded domain. The Dirichlet problem. Monatsh. Math., to appear. Course Notes, UAM (1997), http://www.uam.es/ juanluis.vazquez.

55. VÁzqueZ, J. L. Darcy's Law and the theory of shrinking solutions of fast diffusion equations. SIAM J. Math. Anal., to appear.

56. VÁZqueZ, J. L. Positivity, propagation properties and needles in nonlinear singular diffusion. Preprint, Univ. Autónoma de Madrid.

57. VÁzquez, J. L. One-Dimensional Nonlinear Diffusion. Course Notes, UAM (1999). Book in preparation.

58. Weickert, J. Applications of nonlinear diffusion in image processing and computer vision. Acta Math. Univ. Comenian. (N.S.) 70 (2000), 33-50. Zbl 0988.35070 MR 2002i:68122

59. Weickert, J. \& Benhamouda, B. A semidiscrete nonlinear scale-space theory and its relation to the Perona-Malik paradox. Theoretical Foundations of Computer Vision (Dagstuhl, 1996), F. Solina et al. (eds.), Springer, Wien (1997), 1-10.

60. WitelsKi, T. P. The structure of internal layers for unstable nonlinear diffusion equations. Stud. Appl. Math. 97 (1996), 277-300. Zbl 0860.76087 MR 98g:35099 